\title{
ARTICLE
}

\section{Development of novel rectum dosimeter using OSL sheet with the aim of direct dose measurement of organ dose during brachytherapy}

\author{
Takashi Asahara $^{a^{*}}$, Hiroaki Hayashi ${ }^{\text {a,f }}$, Emi Tomita ${ }^{\text {b,f }}$, Kanako Sakuragawa $^{\mathrm{c}}$, Hiroshi Saegusa ${ }^{\mathrm{c}}$, \\ Yasufumi Shitakubo ${ }^{c}$, Hitoshi Ikushima ${ }^{\text {a,c }}$, Yuki Kanazawa ${ }^{a}$, Yoshiki Mihara ${ }^{a}$, \\ Yoshinori Miyahara $^{\mathrm{d}}$, Tohru Okazakie, Takuya Hashizume ${ }^{\mathrm{e}}$ and Vergil Lorenzo E. Cruz \\ ${ }^{a}$ Graduate School of Health Sciences, Tokushima University, 3-18-15, Kuramoto, Tokushima, 770-8503, Japan; ${ }^{b}$ School of Health \\ Sciences, Tokushima University, 3-18-15, Kuramoto, Tokushima, 770-8503, Japan; 'Tokushima University Hospital, 2-50-1, \\ Kuramoto, Tokushima, 770-8503, Japan; 'Shimane University Hospital, 89-1, Enya, Izumo, Shimane, 693-0021, Japan; \\ ${ }^{e}$ Nagase Landauer, Ltd., C22 gaiku-1, Suwa, Tsukuba, Ibaraki, 300-2686, Japan; ${ }^{f}$ College of Medical, Pharmaceutical and Health \\ Sciences, Kanazawa University, 5-11-80 Kodatsuno, Kanazawa, Ishikawa, 920-0942, Japan
}

\begin{abstract}
During brachytherapy for cancer of uterine cervix using ${ }^{192} \mathrm{Ir}$, organ doses should be managed. In the clinical treatment, the absorbed doses are calculated by a therapeutic planning system, but actual doses are not measured. In order to measure the amount of radiation the rectum is exposed to we have developed a new rectum dosimeter using an optically stimulated luminescence (OSL) sheet. In this study, we aim to propose a calibration procedure. First, we fabricated a novel phantom which consisted of water and acrylic; a radioactive source and an area in which our rectum dosimeter can be inserted. Second, a clinically used therapeutic system was used to calibrate the rectum dosimeter; namely, the doses related to the dosimeters were calibrated using precise calculations. At this time, to obtain a three-dimensional image from the system, a one-beam computed tomography (CT) system was used. In addition, a Monte-Carlo simulation was performed to consider the difference between ideal conditions (100\% water used in the therapeutic planning system) and realistic phantoms. Then, an experiment to calibrate the actual rectum dosimeter was carried out. As the results show, a calibration curve can be determined in the dose region of $0.1-10 \mathrm{~Gy}$ with an accuracy of $15 \%$. Furthermore, absorbed dose of impact of distance between radioactive source and dosimeters was estimated using Monte-Carlo simulation.
\end{abstract}

Keywords: brachytherapy; cervical cancer; organ dose; high dose rate; in-vivo dosimetry; optically stimulated luminescence; direct dose measurement; Monte Carlo simulation; calibration curve

\section{Introduction}

High-Dose-Rate (HDR) brachytherapy with ${ }^{192} \operatorname{Ir}$ is widely used for treatment of uterine cervix cancer, because this treatment often leads to a favorable outcome [1,2]. In HDR brachytherapy, an applicator is fixed in the cervix uteri. Many source dwelling points can be set with the applicator. The radioactive source is remotely inserted into the applicator which enables the medical staff to perform the treatment without radiation exposure. In this method, the absorbed dose of organs at risk, such as rectum, should be properly managed $[3,4]$. Generally, the doses of organs at risk in the patient are estimated by a clinical treatment system [5], and other studies using phantoms were carried out [6]. We consider that measurement of the actual dose to the patient should be performed because there are unknown error factors in the evaluation mentioned above; for

*Corresponding author. Email: takashi.asahara.111@gmail.com example, movement of patient during treatment is not taken into considering.

We have performed a basic study for using optically stimulated luminescence (OSL) dosimeters in the diagnostic region. These dosimeters have good characteristics for dosimetry [7]. Using an OSL sheet which is used in the construction of commercial type OSL dosimeters, we have developed a new rectum dosimeter [8]. This dosimeter consists of Nelaton catheter and the OSL sheet $(1 \mathrm{~cm} \times 2 \mathrm{~cm}$ square). To apply to actual clinical conditions, the value measured with the novel dosimeter needs to be calibrated with absorbed doses.

In this study, we proposed a calibration procedure using Monte-Carlo simulation and performing basic experiments. Then, the accuracy of the dosimeter was evaluated. Additionally, we will discuss the impact of the distance between the radioactive source and dosimeters on the measured dose. 


\section{Materials and methods}

Figure 1 shows the procedure to calibrate our rectum dosimeter. We fabricated a novel phantom to calibrate the dosimeter accurately; the details are described later. Using this phantom calibration experiments were performed with the help of a therapeutic planning system in which an X-ray image was obtained by a cone-beam computed tomography (CT) system used for actual clinical treatment; namely, we calibrated the dosimeter by mimicking the procedure of clinical treatment. In the cone-beam CT system, an ideal phantom consisting of an infinite-volume of water is assumed, and the cone-beam CT X-ray image is used as a guide for scaling; namely, the system can not reflect realistic constructions on calculated dose. The calculation process reported by Task Group 43 American Associate of Physicists in Medicine was adopted for clinical treatment [9]. The phantom fabricated in this study consists of acrylic and water, therefore, we calculated the difference between ideal and actual phantoms using Monte-Carlo simulation code, EGS5 [10]. In this study, we constructed a calibration curve which was defined as a relationship between absorbed dose (X axis) and response of OSL dosimeter (Y axis).

The detail of the novel phantom is represented in Figure 2. The outer size of the acrylic phantom was 100 $\mathrm{mm} \times 100 \mathrm{~mm} \times 100 \mathrm{~mm}$. It has three insert holes; center, left, and right holes which can be used to insert a radioactive source $\left({ }^{192} \mathrm{Ir}\right)$, rectum dosimeter, and an acrylic rod with a commercial type OSL dosimeter (nanoDot $^{\mathrm{TM}}$, Landauer, Glenwood, Illinois, U.S.A.) implanted, respectively. To check the reliability of our rectum dosimeter, a commercial type nanoDot OSL dosimeter was used as a reference dosimeter. The distance between center and side holes was $30 \mathrm{~mm}$. Water was filled in the phantom.

Our experiments were carried out to obtain a calibration curve of the rectum dosimeter. In order to install three-dimensional scaling of our phantom, we obtained the image by cone-beam CT system and then, therapeutic planning system was used to calculate dose distribution in the phantom. To achieve accurate calibration, we corrected the difference between ideal and realistic phantoms using EGS5 simulation [11]. In the simulation, we assumed ideal and actual phantoms, and a correction factor which can convert the ideal dose to the actual dose was derived. The absorbed dose was determined by the planning system taking into consideration the factors mentioned above. Our rectum dosimeter and nanoDot OSL dosimeter were inserted into the phantom and irradiated with the following condition. Absorbed doses were set at 0.1, 0.2, 0.4, 0.6, $0.8,1.0,2.0,4.0,6.0,8.0$, and $10 \mathrm{~Gy}$. We used ${ }^{192} \mathrm{Ir}$ as the source with $300 \mathrm{GBq}$ which was remotely operated by a clinical system (microSelectron HDR-V2 ${ }^{\mathrm{TM}}$, Elekta, Stockholm, Sweden). Typical irradiation rate was approximately $1 \mathrm{~Gy} / 1 \mathrm{~min}$; source dwelling times were precisely determined by the system taking into consideration radioactive decay of ${ }^{192} \mathrm{Ir}$.
After irradiating, response counts of these OSL dosimeters, were analyzed by reading device (microStar $^{\mathrm{TM}}$, Landauer, Glenwood, Illinois, U.S.A.). Here, because OSL dosimeters have different intrinsic efficiencies $(\varepsilon)$, we divided counts by $\varepsilon$. The response of OSL dosimeter was defined as counts $/ \varepsilon$.

Finally, we analyzed the relationship (calibration curve) between the counts/ $\varepsilon$ and absorbed doses.

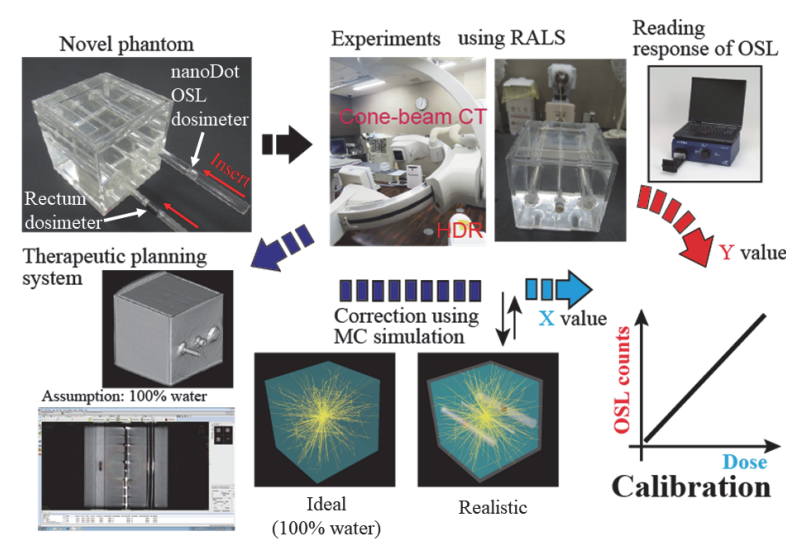

Figure 1. The procedure of calibrating our rectum dosimeter. We fabricated a novel phantom. Exposure dose of the rectum dosimeter was determined by a therapeutic planning system in which the X-ray image obtained by cone-beam CT was used. The difference between the ideal phantom (100\% water) and the actual phantom was considered by the correction factor which was estimated by the Monte-Carlo simulation (see text).

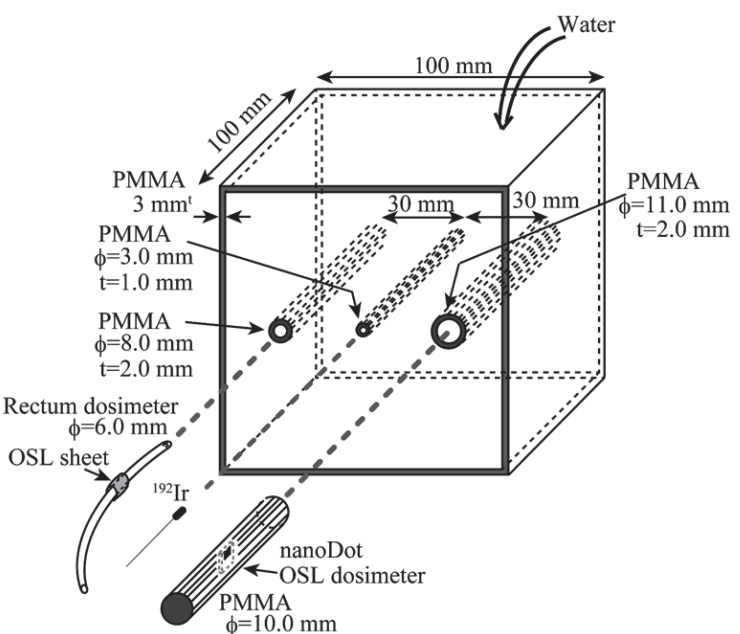

Figure 2. Schematic drawing of a novel phantom. Our phantom consists of a water tank in which there are three insert holes made with acrylic tubing. The radioactive source was inserted into the center hole and dosimeters were inserted into the side holes.

\section{Results and discussion}

\subsection{Calibration curve}

The correction factors which were obtained with actual dose measurements of the phantom study were determined to below as follows: 1.007 for the rectum dosimeter and 1.002 for the nanoDot OSL dosimeter. These correction factors were nearly equal to 1 . This fact means that our phantom was close to an ideal phantom.

The upper figure in Figure 3 shows a calibration curve of the rectum dosimeter and nanoDot OSL 
dosimeter. The horizontal axis indicates absorbed dose in the experiment, we set exposure doses using a therapeutic planning system and multiplied the correction factors mentioned above. The blue and red circles are the data measured using the rectum dosimeter and nanoDot OSL dosimeter, respectively.

The data in a dose region of 0.1-1 Gy were analyzed and a fitted line of $Y=8335 X$ was obtained. The lower figure represents the deviation from the fitted line. It is clear that the data gradually increase over 1 Gy. Therefore, another fitted line was applied to the data over $1 \mathrm{~Gy}$; as represented in the upper plot, the calibration curve for the higher dose region was determined to be $Y=-15856+92492 X$.
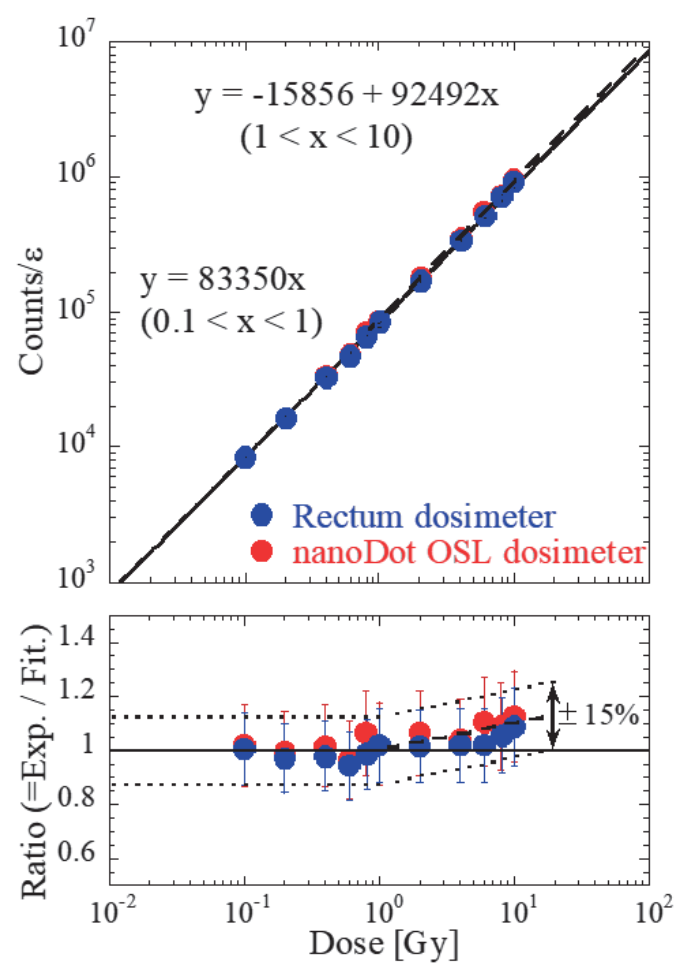

Figure 3. Calibration curve of the rectum dosimeter (upper), and the deviation (lower). The accuracy of the dosimeter was estimated to be $15 \%$.

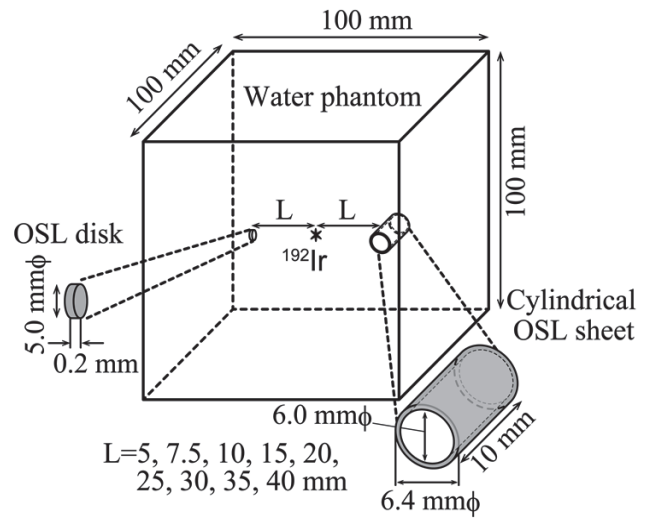

Figure 4. Schematic drawing of simulation condition to evaluate the impact of distance between radioactive source and dosimeters. An OSL disk shown on the left and cylindrical OSL sheet on the right, indicate ideal and actual dosimeters, respectively.
Using the lower plot, we estimated the accuracy of determined dose. It is well known that uncertainty of intrinsic detection efficiency of the nanoDot OSL dosimeter is at most 5\% [7]. We have performed other experiments concerning the evaluation of angular dependence, and the accuracy of angular dependence was estimated to be $10 \%$. For evaluation of the present experiment, we preliminarily added the uncertainty of $15 \%$. In the lower plot, the range of $+/-15 \%$ is identified by dashed lines and all experimental data is included in that range.

We evaluated that the accuracy of the calibration for our rectum dosimeter and found it to be at most $15 \%$.

\subsection{Limitation to apply our dosimeter}

As described above, our rectum dosimeter was cylindrical in form, and the averaged value of the cylindrical detection region was adopted as the experimental value. This averaged value seems to be the same with point dose at the center position, when the dosimeter was used under the condition in which the distance between radioactive source and dosimeter was sufficiently long. In this section, we confirm the limitation of our rectum dosimeter using EGS5 code.

Figure 4 shows the condition of simulation. A water phantom was assumed, two types of dosimeters (small OSL disk and cylindrical OSL sheet) were placed in left and right sides, respectively. The sizes of dosimeters are represented in the figure. The distance (L) between the radioactive source and dosimeters was set at 5, 7.5, 10, $15,20,25,30,35$, and $40 \mathrm{~mm}$. Then, absorbed doses for the small OSL disk and cylindrical OSL sheet were calculated. The small OSL disk can be regarded as a point detector and a realistic rectum dosimeter was reproduced by using a cylindrical OSL sheet.
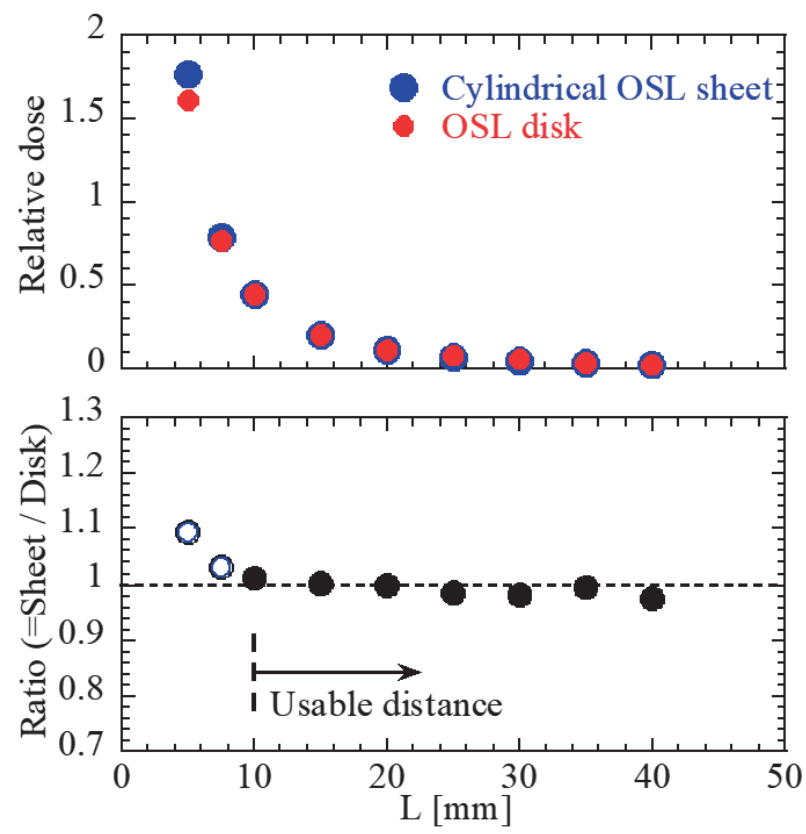

Figure 5. Simulated result to evaluate impact of distance on measured doses. Upper graph shows relative doses for OSL disk and cylindrical OSL sheet. Lower graph indicates ratio of calculated doses. 
The result of the simulation is shown in the upper graph of Figure 5. The horizontal axis represents L, and the vertical axis shows relative doses of dosimeters. The red and blue circles were the doses of the OSL disk and cylindrical OSL sheet, respectively. The lower figure shows the ratio of the doses between cylindrical OSL sheet and OSL disk. When L became larger than $10 \mathrm{~mm}$, the dose measured with the cylindrical OSL sheet (mean value) was consistent with the dose measured with OSL disk. On the other hand, when $\mathrm{L}$ was less than $10 \mathrm{~mm}$, the cylindrical OSL sheet cannot be regarded as a point detector. In our experiment for obtaining the calibration curve, the distance between the radioactive source and dosimeters was set at $30 \mathrm{~mm}$, therefore the rectum dosimeter can be calibrated properly. It is important that the rectum dosimeter should be used in a clinical study under the above-mentioned limitation.

\section{Conclusion}

In order to apply to our dosimeter to actual dose measurement during brachytherapy, the rectum dosimeter was calibrated using a clinical therapeutic system. Then, we determined a precise calibration curve by dividing the curve in two dose regions (0.1-1 Gy and 1-10 Gy). The accuracy of our calibration curve was estimated to be $15 \%$. Additionally, the limitation of our dosimeter was evaluated by means of Monte-Carlo simulation. The usable distance between a radioactive source and dosimeters was estimated to be over $10 \mathrm{~mm}$. Under real clinical conditions, this condition may be achieved. Therefore, we obtained a prospective dosimeter capable of measuring the actual radiation dose to rectum during brachytherapy of the cervix uteri.

\section{References}

[1] S. Nag, B. Erickson, B. Thomadsen, C. Orton, J.D. Demanes and D. Petereit, The American Brachytherapy Society recommendations for high-dose-rate brachytherapy for carcinoma of the cervix, International Journal of Radiation Oncology Biology Physics. 48 (1) (2000), pp. 201-211. DOI: 10.1016/S0360-3016(00)00497-1

[2] H. Ikushima, Y. Takegawa, K. Osaka, S. Furutani, K. Yamashita, T. Kawanaka, A. Kubo, T. Kudoh, and H. Nishikawa, Radiation therapy for cervical cancer in the elderly, Gynecologic Oncology. 107 (2) (2007), pp. 339-343. DOI: 10.1016/j.ygyno. 2007.07.058

[3] K. Kirchheiner, R.A. Nout, J.C. Lindegaard, C. Haie-Meder, U. Mahantshetty, B. Segedin, I.M. Jürgenliemk-Schulz, P.K. Hoskin, B. Rai, W. Dörr, C. Kirisits, S.M. Bentzen, R. Pötter and K. Tanderup, Dose-effect relationship and risk factors for vaginal stenosis after definitive radio(chemo)therapy with image-guided brachytherapy for locally advance cervical cancer in the EMBRANCE study, Radiotherapy and Oncology. 118 (1) (2016), pp. 160-166. DOI: 10.1016/j.radonc.2015.12.025

[4] I. Barillot, J.C. Horiot, P. Maingon, G. Truc, G.
Chaplain, J. Comte and J.P. Brenier, Impact on treatment outcome and late effects of customized treatment planning in cervix carcinomas: baseline results to compare new strategies, International Journal of Radiation Oncology Biology Physics. 48 (1) (2000), pp. 189-200. DOI: 10.1016/S0360-3016 (00)00556-3

[5] N. Wachter-Gerstner, S. Wachter, E. Reinstadler, C. Fellner, T.H. Knocke, A. Wambersie and R. Pötter, Bladder and rectum dose defined from MRI based treatment planning for cervix cancer brachytherapy: comparison of dose-volume histograms for organ contours and organ wall, comparison with ICRU rectum and bladder reference point, Radiotherapy and Oncology. 68 (3) (2003), pp. 269-276. DOI: 10.1016/S0167-8140 (03)00189-0

[6] F. Araki, T. Kouno, T. Ohno, K. Kakei, F. Yoshiyama and S. Kawamura, Measurement of absorbed dose-to water for an HDR ${ }^{192}$ Ir source with ionization chambers in a sandwich setup, Medical Physics. 40 (9) (2013), pp. 092101-8. DOI: $10.1118 / 1.4816673$

[7] K. Takegami, H. Hayashi, K. Yamada, Y. Mihara, N. Kimoto, Y. Kanazawa, K. Higashino, K. Yamashita, F. Hayashi, T. Okazaki, T. Hashizume and I. Kobayashi, Entrance surface dose measurement using a small OSL dosimeter with a computed tomography scanner having 320 rows of detectors, Radiological Physics and Technology. 10 (1) (2017), pp. 49-59. DOI: 10.1007/s12194016-0366-1

[8] E. Tomita, H. Hayashi, T. Asahara, K. Sakuragawa, Y. Shitakubo, H. Saegusa, H. Ikushima, Y. Kanazawa, S. Goto, T. Okazaki, T. Hashizume and V.L.E. Cruz, Direct radiation dose measurement of rectum during high-dose-rate ${ }^{192} \mathrm{Ir}$ brachytherapy for cervical cancer treatment, Proceedings of the 9th International Symposium on Radiation Safety and Detection Technology (ISORD-9), Jul. 10-14, 2017, Nagoya, Japan, (2017). ISSN: 2185-4823

[9] R. Nath, L.L. Anderson, G. Luxton, K.A. Weaver, J.F. Williamson and A.S. Meigooni, Dosimetry of Interstitial Brachytherapy Sources Report of AAPM Radiation Therapy Committee Task Group 43, Report number: 51, American Association of Physicists in Medicine (1995).

[10]H. Hirayama, Y. Namito, A.F. Bielajew, S.J. Wilderman and W.R. Nelson, THE EGS5 CODE SYSTEM, KEK Report number: 2005-8, High Energy Accelerator Research Laboratory (2005).

[11]L.Beaulieu, A.C. Tedgren, J.F. Carrier, S.D. Davis, F. Mourtada, M.J. Rivard, R.M. Thomson, F. Verhaegen, T.A. Wareing and J.F. Williamson, Report of the Task Group 180 on model-based dose calculation methods in brachytherapy beyond the TG-43 formalism: Current status and recommendations for clinical implementation, Medical Physics. 39 (10) (2012), pp. 6208-6236. DOI: $10.1118 / 1.4747264$ 